\title{
Outcome of Kienböck's disease in twelve cases: a mid-term follow-up study
}

\author{
Ravi Gupta ${ }^{1}$, MS, DNB, Manish $\underline{\text { Pruthi }}^{1}$, MS, DNB, Amit $\underline{K u m a r}^{1}$, MS, Sudhir $\underline{\text { Garg }}{ }^{1}$, MS
}

INTRODUCTION No single study has established the superiority of one treatment of Kienböck's disease over the other. Pooled outcome data is presently considered the best way to add to the knowledge and understanding of Kienböck's disease.

METHODS A total of 12 patients ( 9 male and 3 female) with Kienböck's disease were included in the present case series. The mean age of the 12 patients was 28 years. One patient presented in Lichtman stage I, five in Lichtman stage II, five in Lichtman stage IIIa, and one in Lichtman stage IV. Univariate and multivariate analyses of the obtained data were performed to identify any correlations.

RESULTS The mean follow-up time was 62 months, and the mean modified Mayo wrist score improved from the preoperative value of 29.5 to the final value of 89.6. Lichtman stage at presentation showed moderate positive correlation with the duration of symptoms $(r=0.56)$, and a strong negative correlation with the preoperative and final modified Mayo scores ( $r=-0.89$ and $r=-0.77$, respectively). The final modified Mayo score showed moderate negative correlation with the duration of the symptoms $(r=-0.55)$. There was a significant difference in the preoperative modified Mayo scores of patients who presented in stage II and those of patients who presented in stage IIla $(p=0.03)$. However, the difference in the final modified Mayo scores of the patients in these stages was not significant $(p=0.14)$.

CONCLUSION Lichtman's stage is moderately related to the duration of symptoms, suggesting natural progression of the disease. The final outcomes of stages II and IIla were the same irrespective of the surgical treatment (radial shortening and/or vascularised bone grafting).

Keywords: Kienböck's disease, lunate, wrist

\section{INTRODUCTION}

Kienböck's disease was first described by Robert Kienböck in 1910. He postulated that the disease is caused by disturbed nutrition of the lunate. ${ }^{(1,2)}$ Since its first description in 1910, numerous studies have been published on the various aetiological factors of the disease. Hulten correlated the disease with negative ulnar variance, ${ }^{(3)}$ Lee $^{(4)}$ and Gelberman et al ${ }^{(5)}$ correlated it with arterial supply of the lunate, and Schiltenwolf et al correlated it with obstruction of venous outflow. ${ }^{(6)}$ The current consensus on the aetiological factors of this disorder favours a multifactorial origin. $^{(7)}$

Treatment of Kienböck's disease should focus on relieving pain, halting disease progression and improving function. Various standard modalities are used to treat this disease, including nonsurgical management, vascularised bone graft (VBG), joint levelling procedures, intercarpal arthrodesis, proximal row carpectomy and total wrist arthrodesis. In a landmark paper published in 1977, Lichtman et al described four progressive radiological stages of the disease, thus providing a scale that could be used to compare conditions at presentation, progression in untreated cases and outcomes of different interventions. ${ }^{(8)}$ Based on the current understanding of Kienböck's disease, treatment that addresses the pathology (i.e. lunate load/avascularity in early stages and carpal instability in late stages) is recommended.(7) However, there is no strong evidence in the literature regarding the role of different treatment methods in various stages of the disease.
Since 2004, our centre has been treating patients with Kienböck's disease based on their Lichtman stage at presentation. Herein, we present the outcomes of our patients, who received treatment consistent with the current recommendations.

\section{METHODS}

The present study consists of 12 cases of Kienböck's disease that were treated at our centre over a seven-year period (from October 2004 to October 2011); two cases had earlier been excluded, as the patients were lost to follow-up. All patients were identified via a retrospective review of a prospectively maintained database. The following patient information was recorded: demographic data; time delay between the onset of symptoms and presentation; side of dominance; profession; ulnar variance; Lichtman stage at presentation; modified Mayo wrist score; ${ }^{(9)}$ and type of surgery performed.

Of the 12 included patients (mean age 28 years; range $18-35$ years), 9 (75\%) were male and 3 (25\%) were female. Nine patients had involvement of the dominant side, eight had negative ulnar variance, two had neutral variance and two had positive ulnar variance. One patient presented in Lichtman stage I, five in stage II, five in stage IIla, and one in stage IV. None of the patients presented in stage IIIb (Table I).

The treatment prescribed was based on the stage of the disease and ulnar variance. One patient with stage I disease and neutral ulnar variance received VBG and plaster immobilisation

${ }_{1}^{1}$ Department of Orthopaedics, Government Medical College and Hospital, Chandigarh, India

Correspondence: Dr Manish Pruthi, Assistant Professor, Department of Orthopaedics, Government Medical College and Hospital, Sector 32, Chandigarh 160030, India. manishpruthi@gmail.com 
Table I. Characteristics of patients in the present study $(n=12)$.

\begin{tabular}{|c|c|c|c|c|c|c|c|c|c|c|}
\hline Case & $\begin{array}{l}\text { Age } \\
\text { (yr) }\end{array}$ & Gender & $\begin{array}{l}\text { Affected } \\
\text { side }\end{array}$ & $\begin{array}{c}\text { Side of } \\
\text { dominance }\end{array}$ & $\begin{array}{l}\text { Ulnar } \\
\text { variance }\end{array}$ & Stage & $\begin{array}{c}\text { Preop } \\
\text { Mayo score }\end{array}$ & Treatment & $\begin{array}{c}\text { Final Mayo } \\
\text { score }\end{array}$ & $\begin{array}{c}\text { Duration of } \\
\text { follow-up (mth) }\end{array}$ \\
\hline 1 & 18 & M & $\mathrm{R}$ & $\mathrm{R}$ & Negative & IIIa & 25 & Radial shortening & 90 & 96 \\
\hline 3 & 20 & M & $\mathrm{R}$ & $\mathrm{R}$ & Positive & IV & 15 & Arthrodesis & 60 & 84 \\
\hline 4 & 30 & $\mathrm{~F}$ & $\mathrm{~L}$ & $\mathrm{R}$ & Neutral & I & 40 & VBG and plaster immobilisation & 100 & 72 \\
\hline 5 & 35 & M & $\mathrm{L}$ & $\mathrm{L}$ & Negative & IIIa & 20 & Radial shortening & 90 & 72 \\
\hline 6 & 24 & M & $\mathrm{R}$ & $\mathrm{R}$ & Negative & II & 35 & Radial shortening + VBG & 85 & 65 \\
\hline 7 & 32 & $\mathrm{~F}$ & $\mathrm{~L}$ & $\mathrm{R}$ & Neutral & IIIa & 20 & VBG + temporary SC fixation & 85 & 62 \\
\hline 8 & 35 & M & $\mathrm{L}$ & $\mathrm{R}$ & Negative & II & 30 & Radial shortening + VBG & 100 & 62 \\
\hline 9 & 32 & M & $\mathrm{R}$ & $\mathrm{R}$ & Positive & II & 40 & VBG + temporary SC fixation & 100 & 60 \\
\hline 10 & 27 & M & $\mathrm{R}$ & $\mathrm{R}$ & Negative & II & 40 & Radial shortening + VBG & 95 & 52 \\
\hline 11 & 35 & $\mathrm{~F}$ & $\mathrm{R}$ & $\mathrm{R}$ & Negative & IIIa & 25 & Radial shortening + VBG & 90 & 22 \\
\hline
\end{tabular}

F: female; L: left; M: male; R: right; SC: scaphocapitate; VBG: vascularised bone graft
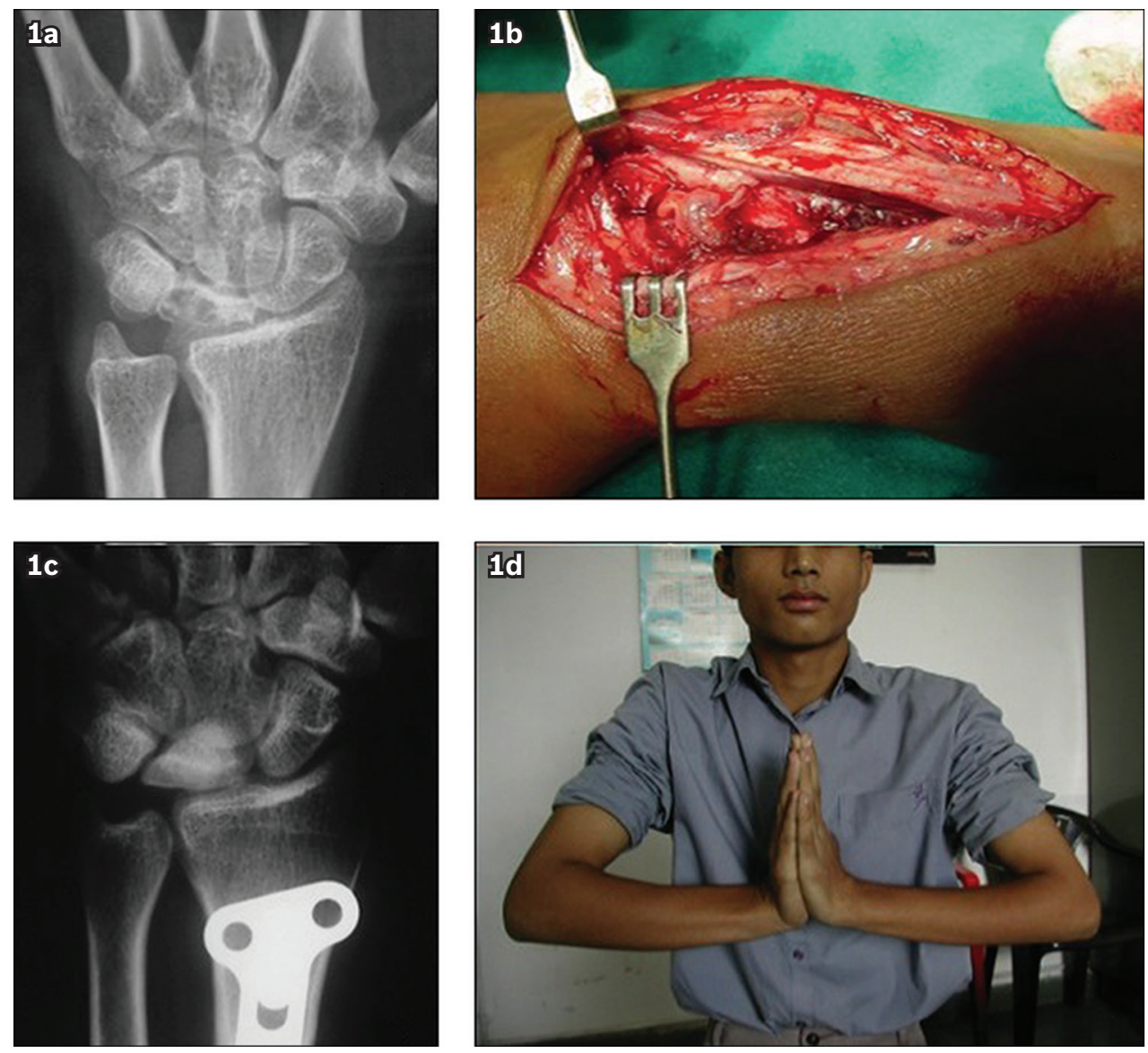

Fig. 1 A patient with stage Illa Kienböck's disease. (a) Preoperative radiograph of the wrist; and (b) intraoperative photograph of the wrist after the vascularised bone graft was impacted into the lunate. (c) Radiograph and (d) photograph taken at follow-up four years after radial shortening and vascularised bone graft show revascularisation of the lunate and good clinical function of the wrist, respectively.

for three months. Out of the ten patients with stage II/IIla disease, eight had negative ulnar variance and were treated with either radial shortening alone $(\mathrm{n}=3)$ or radial shortening combined with VBG $(n=5)$ (Fig. 1). For two patients, one with neutral ulnar variance and the other with positive ulnar variance, VBG and temporary scaphocapitate fixation with two 1.5-mm K-wires were performed; the K-wires were removed after three months. Wrist arthrodesis was performed in one stage IV patient who had a positive ulnar variance (Table I). A single surgeon performed all the surgeries. All preoperative and final radiographs were evaluated for radiological signs of vascularisation, including decrease in sclerosis and restoration of the contour of the lunate.

Univariate and multivariate analyses of data were performed to detect any correlation between Lichtman stage at presentation and the duration of symptoms, side of dominance, ulnar variance and modified Mayo wrist score at presentation. All statistical 
analyses were performed using the Statistical Package for the Social Sciences version 13.0 for Windows (SPSS Inc, Chicago, IL, USA). Correlation of the final outcome with variables such as age, gender, Lichtman stage, side of dominance, ulnar variance, type of surgical procedure and modified Mayo wrist scores at presentation was also analysed. Results were analysed to detect whether there was any difference in the outcomes of patients who underwent vascularised pedicle bone grafting and those who underwent joint levelling surgeries alone. Since treatment options are most uncertain in patients with stages II and IIla disease, the modified Mayo scores of these patients at presentation and final follow-up were analysed separately.

\section{RESULTS}

In the present study, the mean duration of follow-up and mean duration of symptoms were 62 (range 18-96) months and 16 (range 6-36) months, respectively. The mean preoperative modified Mayo wrist score at the time of presentation was 29.58 (range 15-45). The mean modified Mayo score improved from 29.58 to 89.58 at final follow-up, with excellent outcomes observed in eight patients, good outcomes in three patients and poor outcome in one patient. The patient who had poor outcome had presented with stage IV Kienböck's disease and undergone wrist arthrodesis.

The mean modified Mayo wrist score of the patients who were treated with $\mathrm{VBG}$, in combination with other procedures or alone, $(n=8)$ improved from an average preoperative score of 31.3 to an average final score of 92.5 , while that for patients treated with joint levelling surgeries $(n=3)$ improved from an average preoperative score of 30.0 to an average final score of 91.7. The difference between pre- and postoperative scores for both groups was, however, not statistically significant (Mann-Whitney $U$ test, $p=0.916$ ). Further analysis of the ten patients who presented with stages II and IIla Kienböck's disease showed no significant difference between the final outcomes of joint levelling surgery and VBG (Wilcoxon signed rank test; $p=0.125$ for stage II and $p=0.173$ for stage IIIa). For patients treated with VBG, the mean modified Mayo score of stage II patients $(n=4)$, improved from 36.3 to 95.0 , while that of stage IIla patients $(n=3)$ improved from 21.7 to 86.7 . The difference between the preoperative mean scores of stages II and IIla patients was found to be significant $(p=0.03)$, whereas the difference between the mean final modified Mayo scores of stage II and stage IIla patients was not $(p=0.14)$.

The Lichtman stage at presentation showed moderate positive correlation with the duration of symptoms $(r=0.56)$ and a strong negative correlation with the preoperative modified Mayo score $(r=-0.89)$. No significant correlation was found between the Lichtman stage at presentation and other variables, including hand dominance, age, gender and ulnar variance. The final modified Mayo score showed moderate negative correlation with the duration of symptoms $(r=-0.57)$ and strong negative correlation with the Lichtman stage at presentation $(r=-0.772)$. However, it did not show any significant correlation with age, gender, hand dominance, ulnar variance or type of surgery.
All the patients who underwent VBG showed radiological evidence of revascularisation (Fig. 1). Loss of sclerosis and prevention of collapse was taken as the criteria for revascularisation. In the present study, we did not encounter any complications related to the osteotomies or placement of hardware.

\section{DISCUSSION}

In the early stages of Kienböck's disease (i.e. stages I-IIla), the severity of radiological changes in the lunate does not always correlate with the symptoms. ${ }^{(10,11)}$ There are various treatment modalities that address the different pathologies of this disease. A recent meta-analysis, which evaluated the various treatment modalities for Kienböck's disease, showed that subjective pain improved regardless of treatment type, whereas objective improvement in grip strength and range of movement occurred only in patients who received either joint levelling surgeries or VBG, and not in those who had nonsurgical treatments. ${ }^{(12)}$ Joint levelling surgeries have previously been shown to be effective in treating Kienböck's disease with negative ulnar variance. ${ }^{(13,14)}$ In the present study, joint levelling surgeries similarly proved to be effective in all the patients who received this modality of treatment.

As the primary pathology of Kienböck's disease is thought to involve the loss of vascularity, VBG would be expected to improve the local biology. ${ }^{(15,16)}$ However, the role of VBG as the optimum treatment modality has yet to be fully established. ${ }^{(7,15)}$ In Moran et al's study, which consisted of 26 patients with 11 years of follow-up, revascularisation was observed in $71 \%$ of cases. ${ }^{(17)}$ Similar results were observed in Mathoulin and Wahegaonkar's study, which consisted of a series of 22 patients, $77 \%$ of whom showed evidence of revascularisation. ${ }^{(18)}$ In the present study, all the patients who underwent VBG $(n=8)$ showed radiologic evidence of revascularisation (Fig. 1). The modified Mayo wrist score of those who underwent vascularised pedicle bone grafting, however, had similar improvements as those treated with joint levelling surgery (radial shortening), excluding the sole patient who underwent arthrodesis. A literature review by Mehrpour et al suggested that in the early stages (stages I and II) of Kienböck's disease, other techniques (e.g. core decompression of the lunate) are also able to produce improvements that are comparable to joint levelling surgeries. ${ }^{(19)}$ In the present study, we did not find any significant correlation between the Lichtman stage at presentation and age, gender, hand dominance, ulnar variance or type of surgery. These variables also did not show any significant correlation with the final outcome of the disease. There is currently a lack of information in the literature regarding the correlation between age, gender, hand dominance and ulnar variance with the Lichtman stage at presentation and the final outcome of the disease.

The treatment options are traditionally thought to be most uncertain in stages II and IIla of the disease. In the present study, although there was a significant difference between the preoperative modified Mayo scores of patients presenting in Lichtman stage II and patients presenting in stage IIla $(p=0.03)$, 
the difference in the final modified Mayo scores of these two groups of patients was not significant $(p=0.14)$. This suggests that the outcome in Kienböck's disease may not differ significantly as long as carpal alignment is maintained.

The main limitation of the present study is its small sample size. As the incidence of Kienböck's disease is low, pooled data from various institutions that use similar treatment protocols will help to further enhance our understanding of the outcome of this disease. Also, magnetic resonance imaging, which could have been employed to better assess the status of revascularisation, was not used in the present study. Another limitation is the retrospective nature of the study. In addition, there was a lack of consistency in the surgical treatment of patients, even among patients with the same stage of disease. The lack of a standardised radiographic technique used during follow-up visits, which is a mandatory prerequisite to assess the exact gain in height and restoration of contour of the lunate, is another limitation of the present study. For better radiographic evaluation, our institution has now started following a standard regimen of radiography, involving dedicated musculoskeletal radiologists, with a view to obtaining a more accurate evaluation of parameters such as gain in height and contour of the lunate.

In conclusion, although the present study was a small case series, we found that joint levelling surgeries are as effective as VBG in stages II and IIla Kienböck's disease. Therefore, a less morbid procedure such as radial shortening may suffice for the treatment of Kienböck's disease with negative ulnar variance. We also observed that the final outcomes of Lichtman's stages II and IIla Kienböck's disease are not significantly different, as long as carpal alignment is maintained.

\section{REFERENCES}

1. Peltier LF. The classic. Concerning traumatic malacia of the lunate and its consequences: degeneration and compression fractures. Privatdozent Dr. Robert Kienböck. Clin Orthop Relat Res 1980; 149:4-8.
2. Staåhl F. On lunatomalacia (Kienböck's disease): a clinical and roentgenological study, especially on its pathogenesis and late results of immobilization treatment. Acta Chir Scand (Suppl) 1947; 126:1-133.

3. Hulten O. Über anatomische variationen der handgelenkknochen. Acta Radiol 1928; 9:155-168.

4. Lee ML. The intraosseus arterial pattern of the carpal lunate bone and its relation to avascular necrosis. Acta Orthop Scand 1963; 33:43-55.

5. Gelberman RH, Bauman TD, Menon J, Akeson WH. The vascularity of the lunate bone and Kienböck's disease. J Hand Surg Am 1980; 5:272-8.

6. Schiltenwolf M, Martini AK, Eversheim S, Mau H. [Significance of intraosseous pressure for pathogenesis of Kienböck's disease]. Handchir Mikrochir Plast Chir 1996; 28:215-9. German.

7. Lichtman DM, Lesley NE, Simmons SP. The classification and treatment of Kienbock's disease: the state of the art and a look at the future. J Hand Surg Eur Vol 2010; 35:549-54.

8. Lichtman DM, Mack GR, MacDonald RI, Gunther SF, Wilson JN. Kienböck's disease: the role of silicone replacement arthroplasty. J Bone Joint Surg Am 1977; 59:899-908.

9. Slutsky DJ. Outcomes assessment in wrist surgery. J Wrist Surg 2013;2:1-4.

10. Delaere O, Dury M, Molderez A, Foucher G. Conservative versus operative treatment for Kienböck's disease. A retrospective study. J Hand Surg $\mathrm{Br}$ 1998; 23:33-6.

11. Evans G, Burke FD, Barton NJ. A comparison of conservative treatment and silicone replacement arthroplasty in Kienböck's disease. J Hand Surg Br 1986; 11:98-102.

12. Innes L, Strauch RJ. Systematic review of the treatment of Kienböck's disease in its early and late stages. J Hand Surg Am 2010; 35:713-7, 717.e1-4.

13. Almquist EE, Burns JF Jr. Radial shortening for treatment of Kienböck's disease--a 5- to 10-year follow-up. J Hand Surg Am 1982; 7:348-52.

14. Armistead RB, Linscheid RL, Dobyns JH, Beckenbaugh RD. Ulnar lengthening in the treatment of Kienböck's disease. J Bone Joint Surg Am $1982 ; 64: 170-8$.

15. Hori $\mathrm{Y}$, Tamai S, Okuda $\mathrm{H}$, et al. Blood vessel transplantation to bone. J Hand Surg Am 1979; 4:23-33.

16. Kakar S, Giuffre JL, Shin AY. Revascularization procedures for Kienböck's disease. Tech Hand Up Extrem Surg 2011; 15:55-65.

17. Moran SL, Cooney WP, Berger RA, Bishop AT, Shin AY. The use of the $4+5$ extensor compartmental vascularized bone graft for the treatment of Kienböck's disease. J Hand Surg Am 2005; 30:50-8.

18. Mathoulin C, Wahegaonkar AL. Revascularization of the lunate by a volar vascularized bone graft and an osteotomy of the radius in the treatment of Kienböck's disease. Microsurgery 2009; 29:373-8.

19. Mehrpour SR, Kamrani RS, Aghamirsalim MR, Sorbi R, Kaya A. Treatment of Kienböck disease by lunate core decompression. J Hand Surg Am 2011; 36:1675-7. 